Wochen möglich. Das Injektionsintervall in der Erhaltungsphase liegt dann wie gewohnt zwischen vier und acht Wochen. Eine multizentrische, u.a. am Wiesbadener Allergiezentrum durchgeführte klinische Studie mit 201 Patienten bestätigte die Machbarkeit dieses Konzepts: Bei den immunologischen Parametern stiegen die $\mathrm{IgG}_{4}$-Spiegel und die Spiegel an spezifischem IgE-blockie- rendem Faktor innerhalb der ersten zehn Behandlungswochen hochsignifikant gegenüber den Ausgangswerten an.

Aus dem Vehikel $\mathrm{Al}(\mathrm{OH})_{3}$ sei jetzt ein Adjuvans geworden, fasste Klimek zusammen. Beim Einsatz von Avanz ${ }^{\circledR}$ lassen sich so die Vorteile eines intakten Allergens mit den Vorteilen einer kurzen Aufdosierung kombinieren. Der Antrag auf Zulassung von Avanz ${ }^{\circledR}$ für verschie- dene Allergengruppen entsprechend der neuen Therapieallergene-Verordnung wird vorbereitet.

Industriesymposium „Allergiebehandlung heute - neueste Ergebnisse und aktuelle Entwicklungen in der spezifischen Immuntherapie“.4. Gemeinsamer Deutscher Allergiekongress, Berlin, 3.-6. September 2009. Veranstalter: ALK-Abelló, Wedel

\title{
Welche Therapie will der Patient?
}

$F_{\mathrm{d}}^{\mathrm{u}}$ ür Patienten steht die lang anhaltende Wirkung einer spezifischen Immuntherapie (SIT) im Vordergrund. Das gaben drei Viertel der Teilnehmer einer bundesweiten Telefonumfrage im Jahr 2005 an. Knapp die Hälfte der Befragten legte außerdem viel Wert auf eine rasche Wirksamkeit und $41 \%$ auf wenig $\mathrm{Ne}$ benwirkungen, berichtete Priv.-Doz. Dr. Randolf Brehler, Altenberge.

Für die Wirksamkeit der SIT ist eine hohe kumulative Dosis und damit das schnelle Erreichen einer Erhaltungsdosis zwischen 5 und $20 \mu \mathrm{g}$ Majorallergen pro Injektion von Bedeutung. Nur eine hohe Allergendosis induziere Toleranz, betonte Brehler. Bei der so genannten QuickAufdosierung mit Depiquick ${ }^{\circledR}$ wird be- reits am ersten Tag die Erhaltungsdosis erreicht: Es lassen sich dann sechs Maximaldosen in nur fünf Wochen applizieren. Möglich ist dies durch die patentierte Depigmentierung der Allergene, bei der die Allergenität um bis zu 95\% reduziert wird, ohne dass die Immunogenität leidet.

Die Wirksamkeit und Sicherheit der Quick-Aufdosierung belegen bereits mehrere Studien. Brehler selbst hat prospektiv die Sicherheit einer solchen raschen Aufdosierung im Vergleich zur konventionellen dreiwöchigen Aufdosierung mit Depigoid ${ }^{\circledR}$ bei 303 Patienten untersucht. Dabei waren systemische Reaktionen des Grades 1 bei rascher Aufdosierung etwas häufiger, schwere sys- temische Reaktionen traten aber nicht auf. Brehler beurteilte das Verträglichkeitsprofil somit als gleich gut. Was die Wirksamkeit und Sicherheit der SIT mit Depiquick $^{\circledR}$ angeht, so liegen dazu inzwischen Daten von mehr als 1.500 Patienten vor. Eine aktuelle Untersuchung mit Depiquick $^{\circledR}$ wurde im Juni 2009 auf dem europäischen Allergiekongress in Warschau vorgestellt. Depiquick ${ }^{\circledR}$ reduzierte danach bei 72 Patienten mit Rhinokonjunktivitis aufgrund einer Gräserpollenallergie innerhalb von elf Wochen den nasalen Symptomscore im Median signifikant stärker als Plazebo. $\quad f k$

Industriesymposium „Therapie und Abrechnung - was gibt's Neues bei SCIT?". 4. Gemeinsamer Deutscher Allergiekongress, Berlin, 3.-6. September 2009. Veranstalter: Novartis, Nürnberg

\section{Jahre Allergologie im Rückblick}

\footnotetext{
A nlässlich des 50-jährigen Firmenjubiläums von HAL Allergy blickte Prof. Dr. Dr. Johannes Ring auf die Geschichte der Allergologie zurück. Als das Haarlem Allergenen Laboratorium BV im Jahre 1959 in der Nähe von Amsterdam als Servicelabor für die lokalen Universitätskliniken gegründet wurde, konnte niemand ahnen, wie rasant sich die Allergologie in den nächsten Jahrzehnten entwickeln würde. Zu dieser Zeit war das Fach kaum an Universitäten vertreten, die Patientenzahlen waren gering. Heute dagegen gilt Allergie als Volkskrankheit und die Allergologie hat sich zum interdisziplinären Fach mit intensiver Forschungstätigkeit entwickelt.
}
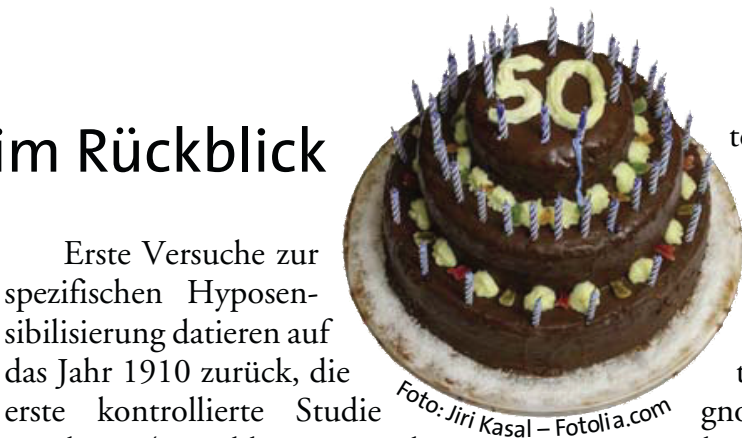

ten, doppelblinden, kontrollierten Studien belegt.

All diese Fortschritte werden dadurch getrübt, dass die Allergologie in Deutschland vor einem Existenzproblem steht: Die Diastik wird kaum mehr erstatwurde 1949 publiziert. In den 1970 er Jahren folgten erste Studien zu Allergoiden, bei denen aufgrund einer chemischen Modifikation der Allergene die gewünschte Erhaltungsdosis mit weniger Injektionen erreicht werden konnte. Die Purethal ${ }^{\circledR}$-Produkte von HAL enthalten chemisch mit Glutaraldehyd modifizierte Allergene, die an Aluminiumhydroxid adsorbiert sind. Wirksamkeit und Verträglichkeit einer Therapie mit Purethal ${ }^{\circledR}$ wurden in randomisier- tet, die Therapie auf die symptomatische Behandlung reduziert, obwohl mit der Hyposensibilisierung eine kausale Option zur Verfügung steht. So forderte Ring eine ausreichende Finanzierung allergologischer Leistungen.

Industriesymposium „50 Jahre $\mathrm{HAL}$ Allergy - 100 Jahre Allergie“. 4. Gemeinsamer Deutscher Allergiekongress, Berlin, 3.-6. September 2009. Veranstalter: HAL, Düsseldorf 